\title{
Wake-promoting actions of median nerve stimulation in TBI-induced coma: An investigation of orexin-A and orexin receptor 1 in the hypothalamic region
}

\author{
YING-JUN ZHONG, ZHEN FENG, LIANG WANG and TIAN-QI WEI \\ Department of Rehabilitation, The First Affiliated Hospital of Nanchang University, Nanchang, Jiangxi 330006, P.R. China
}

Received August 21, 2014; Accepted May 8, 2015

DOI: $10.3892 / \mathrm{mmr} .2015 .3898$

\begin{abstract}
A coma is a serious complication, which can occur following traumatic brain injury (TBI), for which no effective treatment has been established. Previous studies have suggested that neural electrical stimulation, including median nerve stimulation (MNS), may be an effective method for treating patients in a coma, and orexin-A, an excitatory hypothalamic neuropeptide, may be involved in wakefulness. However, the exact mechanisms underlying this involvement remain to be elucidated. The present study aimed to examine the arousal-promoting role of MNS in rats in a TBI-induced coma and to investigate the potential mechanisms involved. A total of 90 rats were divided into three groups, comprising a control group, sham-stimulated (TBI) group and a stimulated (TBI + MNS) group. MNS was performed on the animals, which were in a TBI-induced comatose state. Changes in the behavior of the rats were observed following MNS. Subsequently, hypothalamic tissues were extracted from the rats 6,12 and $24 \mathrm{~h}$ following TBI or MNS, respectively. The expression levels of orexin-A and orexin receptor-1 (OX1R) in the hypothalamus were examined using immunohistochemistry, western blotting and an enzyme-linked immunosorbent assay. The results demonstrated that 21 rats subjected to TBI-induced coma exhibited a restored righting reflex and response to pain stimuli following MNS. In addition, ignificant differences in the expression levels of orexin-A and OXIR were observed among the three groups and among the time-points. Orexin-A and OX1R were upregulated following MNS. The rats in the stimulated group reacted to the MNS and exhibited a re-awakening response. The results of the present study indicated that MNS may be a therapeutic option for TBI-induced coma. The mechanism may be associated with increasing
\end{abstract}

Correspondence to: Professor Zhen Feng, Department of Rehabilitation, The First Affiliated Hospital of Nanchang University, 17th Yong Wai Zheng Street, Nanchang, Jiangxi 330006, P.R. China E-mail: fengzhenly@sina.com

Key words: wake-promoting, median nerve stimulation, traumatic brain injury, coma, orexin-A, orexin receptor 1 expression levels of the excitatory hypothalamic neuropeptide, orexin-A, and its receptor, $\mathrm{OX} 1 \mathrm{R}$, in the hypothalamic region.

\section{Introduction}

A coma is a serious complication, which can occur following traumatic brain injury (TBI) (1). The arousal of a patient from a coma is a priority in improving the functional outcome of the patient, reducing disability and increasing quality of life. Previous evidence has indicated that the central orexinergic/hypocretinergic systems exhibit prominent arousal promoting actions. Rejdak et al (2) described a decreased level of orexin-A in the cerebrospinal fluid of patients following acute brain injury caused by a hemorrhagic stroke. The hypocretin/orexin neuropeptide is synthesized exclusively in the lateral hypothalamus and are involved in several brain functions, including animal survival instincts, and the promotion and maintenance of arousal in animals, a core process in animal behavior (3). The orexinergic neurons not only receive extensive innervations encoding physiological, psychological and environmental cues, but also send final outputs to key arousal-promoting brain areas (3). The arousal-promoting effects of orexin-A act on the promotion of cortical activity and with other neurons associated with wakefulness, including the histaminergic, noradrenergic and serotonergic systems (4). However, whether orexin-A has a wake-promoting effect on pathological conditions, including coma, remains to be elucidated.

There are a number of therapeutic methods in the promotion of arousal of patients in a comatose state. However, the effects are not definite. Electrical stimulation (ES) has been used as a therapeutic method in physical therapy for decades (5). Common types of ES involve deep brain stimulation, cervical epidural spinal cord stimulation and peripheral nerve stimulation. Median nerve stimulation (MNS), a form of peripheral nerve stimulation, has been reported to be effective, which has also been used for the treatment of post-stroke, post-trauma, hypoxic states, and imaging and laboratory measures, including electroencephalography (EEG), single-photon emission computed tomography (SPECT) and catecholamine metabolism have demonstrated clinical improvement in patients treated with ES, compared with those without ES (6-8). The mechanisms may involve activation of the neuro-endocrine system to improve functioning following 
traumatic cerebral damage through peripheral routes to central areas. It is hypothesized that the peripheral stimuli connect with the ascending reticular activating system (ARAS), which further connects with intralaminar nuclei of the thalamus, stimulating cortical layer 1 and enhancing arousal $(7,9)$.

MNS may be an effective method of wake-promotion for patients in a TBI-induced comatose state, which may act to activate associated arousal systems, including the orexinergic system and the upregulation of orexin-A and orexin receptor-1 (OX1R) in the hypothalamus. The present study aimed to investigate the potential mechanisms underlying the arousal-promoting action of MNS and examine the key role of orexin-A in promoting arousal from a comatose state.

\section{Materials and methods}

Animalmodel.A total of 100 male/female adult Sprague-Dawley rats weighing 250-300 g were used in the present study. The rats were obtained from the Institute of Laboratory Animals at Nanchang University (Nanchang, China). All rats were housed in the Laboratory Animal Center of the First Affiliated Hospital of Nanchang University and were maintained in an air-conditioned room with a 12-h light/dark cycle, fed a standard diet and with access to water ad libitum. The protocol was approved by the Local Animal Research Committee and was performed in accordance with the Chinese Association for Accreditation of Laboratory Care and National Institutes of Health (Bethesda, MD, USA) guidelines. The ethical approval for the present study was obtained from the Ethics Committee of Nanchang University. Due to a series of complications caused by TBI, only 90 surviving rats were involved in the experimental procedures. The animals were divided into the following three groups, each containing 30 rats: i) Control group without any interventions; ii) Sham-stimulated (TBI) group, in which TBI was induced leading to a comatose state; iii) Stimulated (TBI + MNS) group, in which rats were treated with MNS subsequent to a TBI-induced coma. In the present study, the classic 'free fall weight-drop model' was selected, which was developed initially by Allne to model spinal cord injury in 1911. Subsequently, Scott applied the method to investigate brain injury in 1940 and, in 1981, Freeney improved the technique and succeeded in generating a cerebral cortex contusion model in rats (10). The experimental rats were anesthetized using diethyl ether (concentration $\geq 99 \%$ ) inhalation and allowed to breathe air spontaneously. Whilst anesthesiatized, conventional surgical techniques were used to expose the skull via a $5 \mathrm{~mm}$ vertical incision. A cross hit point was marked using a syringe needle at a $2 \mathrm{~mm}$ distance to the left midline and $1 \mathrm{~mm}$ before the coronal suture. A cylindrical impact hammer, weighing $400 \mathrm{~g}$, was dropped at a vertical height of 40-44 cm, along a vertical metal bar, which hit a plastic spacer on the hit-point, which had been marked, causing a concave fracture of the skull. Following injury, the incision was closed, and the animals were disinfected and transferred to a cage. After $1 \mathrm{~h}$, the level of consciousness was classified into six degrees, according to their sensory and motor functions: i) Activities unlimited within the cage; ii) activities decreased. iii) Activities decreased with motor incoordination; iv) righting reflex could be elicited; animals unable to stand. v) righting reflex disappeared; animals able to react to pain; vi) no reaction to pain. Rats, which were classified as degree $\mathrm{v}$ or vi, which lasted at least $30 \mathrm{~min}$, were deemed to be in a comatose state (11) and were used in the following procedures.

$M N S$. The rats in the stimulated group were treated with MNS following TBI when the total duration in a comatose state reached $30 \mathrm{~min}$. This was performed using a low frequency electrical stimulator (ES-420; ITO Physiotherapy \& Rehabilitation, Tokyo, Japan). An acupuncture needle was inserted in the middle of the wrist joint at a depth of $5 \mathrm{~mm}$ and was connected to the stimulator. The parameters were as follows: Frequency of $30 \mathrm{~Hz}$; pulse width of $0.5 \mathrm{~ms}$; electrical current of $1.0 \mathrm{~mA}$; stimulation duration of $15 \mathrm{~min}$. According to the six assessment criteria of consciousness, the behavior and consciousness of the animals were observed and evaluated in the 1-2 $\mathrm{h}$ following completion of MNS. Finally, the rats were returned back to their cages with sufficient food and water until sacrifice.

Tissue extraction. The rats in the stimulated group were sacrificed 6, 12 or $24 \mathrm{~h}$ after MNS, respectively (n=10/time-point), at the same time as the rats in the corresponding control and sham-stimulated groups. Briefly, the rats were sacrificed using 10\% chloral hydrate i.p. (Beijing Solarbio Science \& Technology Co., Ltd., Beijing, China). The head was removed and the hypothalamic tissues were separated on an ice box. The tissues were preserved in liquid nitrogen (Beijing Solarbio Science \& Technology Co., Ltd.). The expression of orexin-A was detected using immunohistochemistry, western blot analysis and ELISA.

Immunohistochemistry. Under deep anesthesia with intraperitoneal chloral hydrate (200 and $20 \mathrm{mg} / \mathrm{kg}$, respectively), the rats were perfused with phosphate-buffered saline (PBS) followed by $4 \%(\mathrm{v} / \mathrm{v})$ paraformaldehyde (Beijing Solarbio Science \& Technology Co., Ltd.). The brains were post-fixed in the same fixative overnight, and then cryoprotected in $20 \%$ sucrose in PBS overnight, following which the tbrains were cut into $40-\mu \mathrm{m}$ coronal sections using a sliding microtome (RM2015; Leica Microsystems, Wetzlar, Germany). For immunostaining, the sections were placed in $0.3 \%(\mathrm{w} / \mathrm{w})$ hydrogen peroxide for $30 \mathrm{~min}$ and then incubated overnight with the primary antibody at room temperature. The primary antibodies included polyclonal rabbit anti-OX1R antibody (cat. no. ab68718; Abcam, Hong Kong, China) and peroxidase-conjugated addinipure goat anti-rabbit IgG (H+L; cat. no. ZB-2301; Beijing Zhongshan Golden Bridge Biotechnology Co., Ltd., Beijing, China). Immunohistochemistry was assessed according to the average gray value and positive cell expression intensity.

Western blotting. The tissue samples were homogenized using a tissue protein extraction kit (cat. no. CW0891; Beijing Cangwei Biotechnology Co., Ltd., Beijing, China). The kit contents included $25 \mathrm{ml}$ tissue protein extraction reagent and $240 \mu 1$ protease inhibitors mixture. The homogenates were centrifuged at $12,000 \mathrm{x}$ g for $10 \mathrm{~min}$ at $4^{\circ} \mathrm{C}$. The supernatants were divided into aliquots and stored at $-80^{\circ} \mathrm{C}$. The concentration of proteins in each sample was determined using a protein assay (Bio-Rad Laboratories, Inc., Hercules, CA, 
USA). Equal quantities of the protein extract $(15 \mu \mathrm{l} ; 7.01 \mu \mathrm{g} / \mu \mathrm{l})$ were added to $10 \%$ SDS-polyacrylamide gels and transferred onto a polyvinylidene difluoride membrane (Beijing Solarbio Science \& Technology Co., Ltd.). Western blotting was performed using a polyclonal rabbit anti-OX1R antibody (1:200; cat. no. ab68718; Abcam) in Tris-buffered saline with Tween-20 (TBS-T) containing 5\% milk. Following an overnight incubation at $4^{\circ} \mathrm{C}$, the membranes were washed three times with TBS-T and incubated for $1 \mathrm{~h}$ at room temperature with peroxidase-conjugated addinipure goat anti-rabbit $\operatorname{IgG}$ (H+L; 1:3,000, cat. no. ZB-2301, Beijing Zhongshan Golden Bridge Biotechnology Co., Ltd.) in TBS-T containing 5\% milk. Subsequently, the blots were treated with chemiluminescence substrate (cat. no. 32109, ECL Plus, GE Healthcare Bio-sciences, Pittsburgh, PA, USA, containing $25 \mathrm{ml}$ luminol/enhancer and $25 \mathrm{ml}$ stable peroxide buffer, and were quantified. Finally, the blots were stripped and reprobed with anti-actin (1:400) to control for loading variations. Quantity One software (version 4.6.2.70; Bio-Rad Laboratories, Inc.) was used to quantify the protein bands. The results were expressed as the mean \pm standard deviation of the ratio of immunoreactivity normalized against $\beta$-actin.

ELISA. The prepared tissue samples were assessed using an ELISA kit for orexin-A (cat. no. cE90607; Wuhan USCN Life Science Inc., Wuhan, China), containing a pre-coated, ready to use 96-well strip plate, standard (liquid; x2), detection reagent $\mathrm{A}(120 \mu \mathrm{l})$, detection reagent $\mathrm{B}(120 \mu \mathrm{l})$, TMB substrate $(9 \mathrm{ml})$, wash buffer $(20 \mathrm{ml})$, plate sealer for 96 wells (x4), standard diluent $(20 \mathrm{ml})$, assay diluent A (6 ml), assay diluent $\mathrm{B}(6 \mathrm{ml})$ and stop solution $(6 \mathrm{ml})$. Following all reagent preparations and set-up of wells for the diluted standard, blank and sample, the standard and sample were diluted, respectively. When the reaction was complete, the samples were analyzed using a microplate reader (Model 680, Bio-Rad Laboratories, Inc.) and measured at $450 \mathrm{~nm}$ immediately. The optical density value of the standard (X-axis) was plotted against the log of the concentration of the standard (Y-axis), and the concentration of orexin-A was calculated.

Statistical analysis. The data of orexin-A and OX1R in the hypothalamus from the different groups and time-points are expressed as the mean \pm standard deviation. The levels of orexin-A and OX1R were analyzed statistically using a $\chi^{2}$ test, homoscedasticity test, one-way analysis of variance, two-way classification analysis of variance and a Kruskal-Wallis H test using SPSS 17.0 (SPSS, Inc., Chicago, IL, USA). For all comparisons, $\mathrm{P}<0.05$ was considered to indicate a statistically significant difference.

\section{Results}

Observation of behavior in rats following MNS. At $1 \mathrm{~h}$ post-MNS, the behavior of rats were re-evaluated using a double-blind technique. The results revealed that only seven rats re-awaked of the 30 sham-stimulated rats (10 degree v; 13 degree vi). However, 21 rats re-awakened in the group of 30 stimulated rats (6 degree v; 3 degree vi), with nine rats remaining in a comatose state.
Immunohistochemistry. Immunoreactivity against OX1R was identified in the cytoplasm of neurons in the hypothalamus (Fig. 1), which regularly lined the circular profile in the cytoplasm. The histological data obtained using the anti-OX1R (Fig. 1) antibodies were similar in terms of the abundance of positive cells, the localization of the positive material and the intensity of staining.

On determining that OX1R was distributed in the cytoplasm of neurons in the hypothalamus, the data from the groups were analyzed using a Kruskal-Wallis $\mathrm{H}$ test. In the hypothalamus, the levels of OX1R in the stimulated group (40.67) were higher, compared with those in the control group (13.75) and sham-stimulated groups $\left(28.08 ; \chi^{2}=27.637\right.$; $\mathrm{P}<0.001)$. No significant difference was observed between the levels of OX1R in each group at the three different time-points.

Western blotting. In the hypothalamus, OX1R exhibited statistically significant differences among the three groups at $6 \mathrm{~h}$ (control<stimulated <sham), $12 \mathrm{~h}$ (sham<control<stimulated), $24 \mathrm{~h}$ (stimulated<control<sham), and among the $6 \mathrm{~h}$ $\left(\mathrm{F}_{2.88}=10.279 ; \mathrm{P}=0.001\right), 12 \mathrm{~h}\left(\mathrm{~F}_{2,88}=11.102 ; \mathrm{P}=0.001\right)$ and $24 \mathrm{~h}\left(\mathrm{~F}_{2.88}=9.347 ; \mathrm{P}=0.002\right)$ time points (Table I and Figs. 2 and 3$)$. As for the difference within the groups, the control group $\left(6<24<1 ; \mathrm{F}_{2,88}=16.674 ; \mathrm{P}<0.001\right)$ and stimulated group $\left(24<6<12 ; \mathrm{F}_{2,88}=79.975 ; \mathrm{P}<0.001\right)$ exhibited statistically significant differences (Table I and Fig. 3). The sham-stimulated group exhibited an increasing trend at 6,12 and $24 \mathrm{~h}$, however, no statistically significant difference was observed. The interaction between the groups and time-points was further analyzed, which revealed that there was an interacting effect between the two factors (groups ${ }^{*}$ time: $\mathrm{F}_{2.88}=14.342 ; \mathrm{P}<0.001$ ).

ELISA. The levels of orexin-A in the different groups and time-points were detected using an ELISA. The level of orexin-A level exhibited a significant increase among the three groups (control<sham-stimulated $<$ stimulated) at $6 \mathrm{~h}(\mathrm{P}<0.001)$ and $12 \mathrm{~h}(\mathrm{P}<0.05$; Table I and Fig. 4). This increasing trend in the three groups was also observed at $24 \mathrm{~h}$, however, without statistical significance. As for the difference of orexin-A within the groups, the three groups all exhibited the same trend $(6<12<24)$. However, only the control group exhibited statistically significant differences $(\mathrm{P}<0.05$; Table I and Fig. 4).

\section{Discussion}

Orexin-A is involved in a variety of physiological functions, including feeding behaviors $(12,13)$, energy homeostasis (14-16), the sleep-wake cycle (17), and the activation of central sympathetic outflow (18). A previous study demonstrated the wake-promoting effects of orexin-A and MNS (4). In addition, orexin-A has been identified as important in the regulation of arousal. Although the numbers of clinical studies investigating the actions of MNS have increased, the exact mechanism remains to be elucidated. Therefore, the present study hypothesized that MNS can be used to promote wakefulness in patients in a comatose state, and that one of the mechanisms was due to its positive effect on orexin-A. There were four aspects arising from this hypothesis, which the present study aimed to investigate. The first involved the association between orexins and wakefulness, the second was 

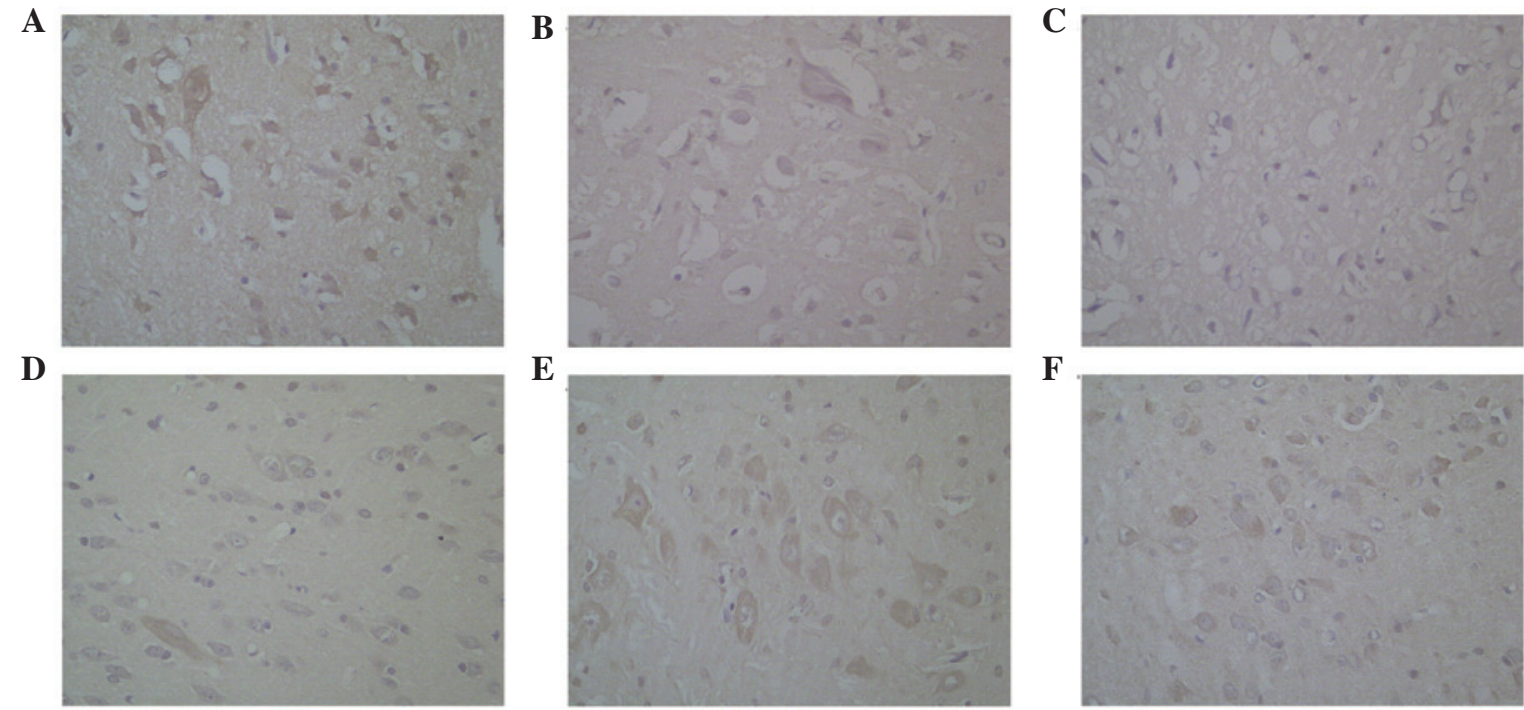

G
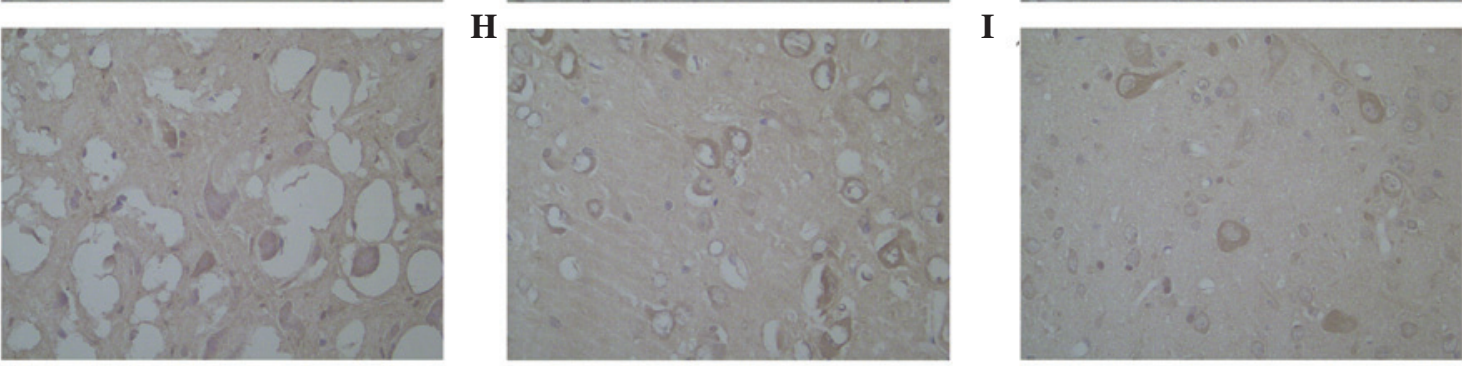

Figure 1. Representative photomicrographs of OX1R-immunoreactivity localization in the hypothalamus. Distribution of OX1R-immunoreactivity in the control group (A) 6, (B) 12 and (C) $24 \mathrm{~h}$ following MNS in the treatment group. Distribution of OX1R-immunoreactivity in the sham-group (D) 6, (E) 12 and (F) $24 \mathrm{~h}$ following MNS in the treatment group. Distribution of OX1R-immunoreactivity in the treatment group (G) 6, (H) 12 and (I) $24 \mathrm{~h}$ following MNS (magnification, $\mathrm{x} 400$ ). Increased OX1R immunostaining was detected within the cytoplasm of neurons in the hypothalamus. OX1R-immunopositive cells were stained and exhibited circular patterns. OX1R, orexin receptor-1; MNS, median nerve stimulation.

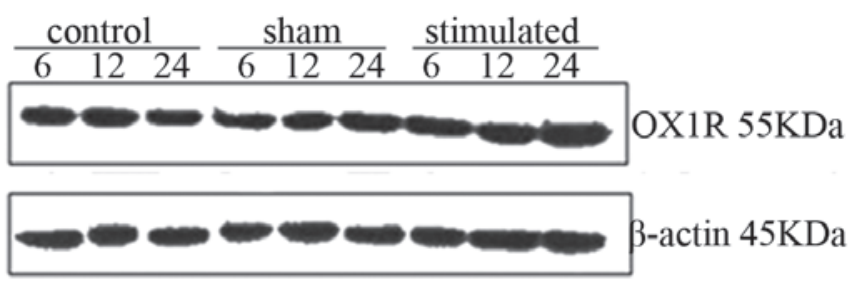

Figure 2. Protein expression of OX1R in the rat hypothalamus. Western blot analysis was performed using anti-serum for OX1R. The upper blots were stripped and re-probed with anti- $\beta$-actin monoclonal antibody to ensure equal loading of proteins in lanes. Lanes 1 and 2 indicate homogenate from a rat hypothlamus. Similar results were obtained from nine separate experiments of identical design. OX1R, orexin receptor-1.

associated with the wake-promoting actions of MNS, the third was associated with the mechanisms of MNS and, finally, the interacting effects were examined.

Orexins, including orexin-A and orexin-B are two excitatory hypothalamic neuropeptides, which are implicated in narcolepsy (19). They are synthesized by neurons situated within the caudal hypothalamus, which projects to the locus coeruleus and other nuclei involved in the regulation of sleep and wakefulness (20). Hypocretin selectively increases dopaminergic neurotransmission within the prefrontal cortex and the shell subregion of the nucleus accumbens, which is also important in cognitive and affective processes (21). Orexin neurons receive abundant input from the limbic system $(22,23)$ and enhance arousal signals in this area. In addition, orexin neurons provide a link between energy homeostasis and vigilance states (24). The data from the present study revealed that higher levels of orexin-A and OX1R following MNS led to arousal behavior in rats. Thus, it was concluded that orexin is an essential neurotransmitter with an arousal-promoting effect. The fluctuation of orexin-A may exert effects on sleep-wakefulness and/or comatose states.

In 1996, peripheral nerve electrical stimulation in the treatment of persistent vegetative states was reported by Yokoyama et al (6). MNS is a form of peripheral nerve electrical stimulation, which is a non-invasive, convenient and economical form of therapy (25). Due to these characteristics, MNS has gradually increased in popularity clinically. It has been reported that right $\mathrm{MNS}$, administered to patients in a comatose state appears to accelerate awakening from a deep coma $(9,26,27)$. Similar to transcutaneous electrical nerve stimulation investigations in Alzheimer's disease, the rationale underlying investigations of coma treatment is that right MNS stimulates the ARAS $(9,28)$. Right MNS has provided promising results in post-treatment improvements, including significantly increased cerebral blood flow and improved electroencephalography (6,7), improved Glasgow Coma Scale scores (9) and, in certain cases, significant or even complete arousal and consciousness $(5,9,26)$. Comparison of pre- and post-treatment SPECT imaging for a series of patients revealed 29 patients $(71 \%)$ with increased cerebral perfusion following 


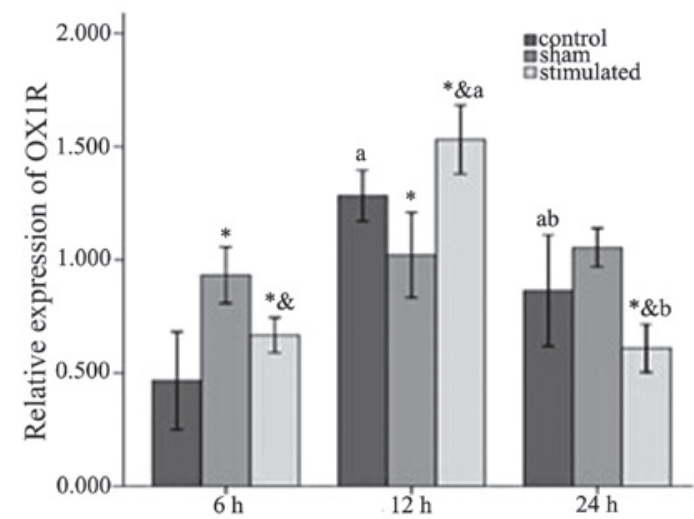

Figure 3. Western blot analysis of OX1R in rat hypothalamic tissues 6,12 and $24 \mathrm{~h}$. Relative protein levels of OX1R were assessed using densitometry. Results were normalized to values obtained for the protein expression of $\beta$-actin. The anti-OX1R antibody detected a band of $55 \mathrm{kDa}$. The intensity of the $55 \mathrm{kDa}$ band was markedly increased and the level of OX1R was highest in the treatment group at $12 \mathrm{~h}$. Statistical analyses consisted of a one-way analysis of variance $(n=10)$. Data are expressed as the mean \pm standard deviation. OX1R within each group was compared at different time-points, ${ }^{\mathrm{a}} \mathrm{P}<0.05,12 \mathrm{~h}$ and/or $24 \mathrm{~h}$, compared with $6 \mathrm{~h},{ }^{\mathrm{b}} \mathrm{P}<0.05,24 \mathrm{~h}$, compared with $12 \mathrm{~h}$. OX1R was compared among the three groups at 6,12 or $24 \mathrm{~h}$. ${ }^{*} \mathrm{P}<0.05$, sham-stimulated group, compared with control group. ${ }^{\&} \mathrm{P}<0.05$, stimulated group, compared with sham-group. OX1R, orexin receptor-1.

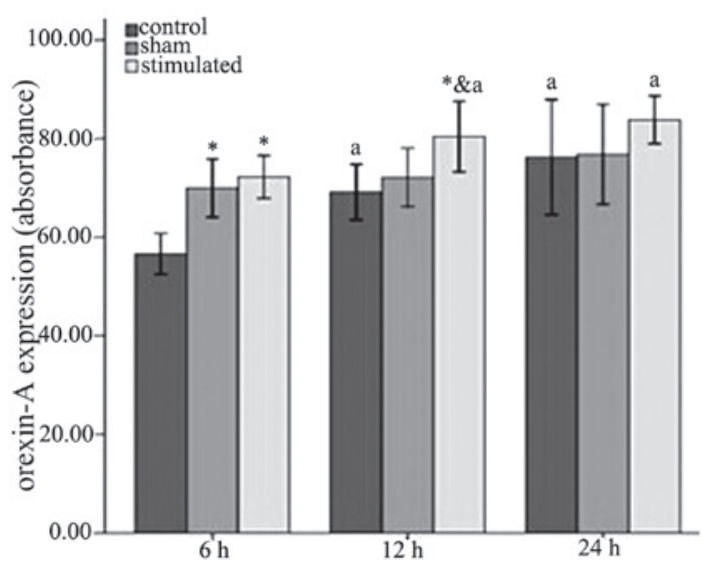

Figure 4. ELISA of orexin-A in the rat hypothalamic tissues. Statistical analyses consisted of a one-way analysis of variance $(n=10)$. Data are expressed as the mean \pm standard deviation. OX1R within each group was compared at three time-points, ${ }^{\mathrm{a}} \mathrm{P}<0.05,12 \mathrm{~h}$ and/or $24 \mathrm{~h}$, compared with $6 \mathrm{~h}$. OX1R was compared among the three groups at the same time points $(6$, 12 or 24 h). ${ }^{*} \mathrm{P}<0.05$, sham-stimulated group, compared with control group; ${ }^{\&} \mathrm{P}<0.05$, stimulated group, compared with sham-stimulated group. OX1R, orexin receptor-1.

MNS, while cerebral perfusion was unchanged in nine patients (22\%) and decreased from baseline in three patients (7\%) (29). It follows that MNS may promote patients in a comatose state to re-awaken and stimulate the central nervous system. In the present study, eight rats in the stimulated group exhibited behavioral actions following MNS, including righting reflex, reactions to pain stimuli and locomotor behavior. However, only one rat recovered motor function with the ability to react to outside stimuli. This was considered a spontaneous recovery, which may have been associated with the degree of injury, individual resistance against damage or somatic function. 
These results inferred that MNS re-awaked animals from an induced comatose state. By contrast, the levels of orexin-A and OX1R were significantly increasing following MNS, which suggested there may be specific connections between MNS, orexin-A and re-awakening from a comatose state.

Although previous investigations have described substantial clinical studies regarding MNS, the exact mechanism of the wake-promoting action of MNS remains to be fully elucidated. The present study aimed to examine the molecular mechanism associated with the neurotransmitter, orexin-A, in the wake-promoting actions of MNS via examining alterations in the levels of orexin-A and OX1R. Several potential mechanisms have been suggested. MNS administration evokes an arousal-promoting effect, possibly through increasing cerebral blood flow and raising levels of dopamine, improving blood supply in ischemic regions, reducing the number of necrotic neurons and protecting neuronal repair and regeneration. Secondly, MNS enhances activities on EEG and electro-neurophysiology assessments. Peripheral electrical signal inputs excite the ARAS and cerebral cortex directly. The ARAS originates in the brain stem reticular formation, more specifically the locus coeruleus and the dorsal raphe nucleus, including the noradrenergic and serotonergic neurotransmitter systems, respectively. In addition, it also has connections with the nucleus basalis of Meynert/cholinergic system $(28,30,31)$. Thirdly, the levels of important neurotransmitters change following MNS. This may be beneficial to relieve patient symptoms and restore corresponding neural functions, including consciousness, mobility and speaking ability. MNS induces the release of acetylcholine from the ARAS, activating the intralaminar nuclei in the thalamus, which have a general excitatory effect on cortical layer 1, and the locus coeruleus, which is located close to the ARAS and releases norepinephrine leading to stimulation of cortical layer 1 . Based on these findings, MNS has been regarded as a 'portal' between the periphery and the central nervous system (28). It has also been reported that feedback from the periphery may contribute to the splitting of circadian rhythms in hamsters (32). In the present study, the expression levels of orexin-A and OX1R exhibited an increasing trend following MNS in the hypothalamus $(\mathrm{P}<0.05)$. As for the three time-points, the levels of OX1R also increased significantly $(\mathrm{P}<0.05)$. The ELISA demonstrated that the expression level of orexin-A in the stimulated group was higher, compared with those in the other two treatment groups and revealed a significant increasing trend between 6,12 and $24 \mathrm{~h}(\mathrm{P}<0.05)$. However, the difference between the control group and the sham-stimulated group was not statistically significant. Thus, the hypothesis that orexin-A and OX1R are upregulated following MNS in TBI-induced-comatose rats was confirmed. This suggested that one of the mechanisms underlying the effect of MNS on comatose states may be attributed to its effect on associated neurotransmitters, including orexin-A.

The results of the present study revealed that, in the hypothalamus, the expression of OX1R was significantly different among three groups at $6 \mathrm{~h}$ (control<stimulated<sham), $12 \mathrm{~h}$ (sham<control<stimulated), $24 \mathrm{~h}$ (stimulated $<$ control $<$ sham) and between the three time-points $(\mathrm{P}<0.05)$. Within the groups, the control $(6<24<12 \mathrm{~h} ; \mathrm{P}<0.001)$ and stimulated group $(24<6<12 \mathrm{~h} ; \mathrm{P}<0.001)$ exhibited statistically significant differences. The sham-stimulated group exhibited an increasing trend at 6, 12 and $24 \mathrm{~h}$, however, no statistically significant difference was observed. By contrast, the level of orexin-A differed significantly among the three groups (control<sham-s timulated<stimulated) at $6 \mathrm{~h}(\mathrm{P}<0.001)$ and $12 \mathrm{~h}(\mathrm{P}<0.05)$. This increasing trend in the three groups was also observed at $24 \mathrm{~h}$, but without statistical significance. In addition, the difference in orexin-A within the three groups exhibited the same trend $(6<12<24 \mathrm{~h})$. However, only the control group exhibited a statistically significant difference $(\mathrm{P}<0.05)$. Based on these findings, the interactive effect between the groups and time-points was analyzed to determine whether these two factors interacted, and an interactive effect was identified between the groups and time-points $(\mathrm{P}<0.001)$. This may be due to its own rhythm of hypothalamic and/or orexin neurons. Lateral hypothalamic orexin-A neurons are rhythmic, innervated by suprachiasmatic nucleus (SCN) efferents and are important components of the reproductive and arousal systems (32). As has been established, the hypothalamus is associated with circadian rhythm. Neurons in the SCN function as the master circadian clock in the brain and are important for hypothalamic rhythm. The SCN regulates orexin neurons so that they are more active during the circadian night, compared with the circadian day. Orexinergic innervation and the expression of genes encoding orexin receptors $\left(\mathrm{OX}_{1}\right.$ and $\left.\mathrm{OX}_{2}\right)$ has also been reported in the mouse $\mathrm{SCN}$, with $\mathrm{OX}_{1}$ being upregulated at dusk (33). Hypocretin deficiency can disturb the circadian control of melatonin release and its temporal association with sleep (34). An investigation of the orange-spotted grouper (Epinephelus coioides) demonstrated that hypothalamic mRNA levels of prepro-orexin were higher in the light phase, compared with the dark phase, and increased significantly at feeding times (35). These observations indicated that orexinergic neurons exhibit a rhythmic activity, and may be active in the day and inactive at night, which are important components of the reproductive and arousal systems (32). Therefore, the present study hypothesized that the rhythm of orexinergic neurons caused the observed interactive effects between the groups and time-points. This may explain why the levels of orexin-A and OX1R changed in each group 6,12 and $24 \mathrm{~h}$ after the experiment.

It is important to note that the present study examined only orexin-A and OX1R in the hypothalamic region. Whether other brain regions are involved in wake-promotion or alterations in the levels of orexin-A and its receptor $24 \mathrm{~h}$ after TBI-induced coma, and how MNS elicits orexin-A increase, remains to be elucidated and requires further investigation. In addition, the present study did not demonstrate whether MNS exerted a direct or indirect effect on orexin-A and OX1R. However, the present study suggested that the level of orexin-A is important in the wake-promoting process, and that the wake-promoting action of MNS was, at least partially, due to the upregulation of orexin-A and OX1R. To overcome these limitations, other brain regions and neurotransmitters associated with orexin-A and/or wakefulness may be considered to determine whether orexin-A acts as an arousal 'switch'. In addition, orexin- $\mathrm{A}^{-1-}$ knock-out animal investigations and the corresponding pathways in ORX neurons require consideration in further investigations to clarify the mechanisms of orexin-A and MNS. 
In conclusion, the present study demonstrated that MNS enhanced the expression levels of orexin-A and OX1R in the hypothalamus, leading to wakefulness from a TBI-induced comatose state, and that orexin-A was important in arousal-promotion. These results suggested that the wake-promoting action of MNS may act on stimulating arousal-associated neurotransmitters, which are released in the hypothalamic region, including orexin-A.

\section{Acknowledgements}

This study was supported by a grant from the Natural Science Foundation of China (grant no. 81260295) and the Natural Science Foundation of Jiangxi Province (grant no. 2013BAB205063).

\section{References}

1. Harvey HH: Reducing traumatic brain injuries in youth sports: youth sports traumatic brain injury state laws, January 2009-December 2012. Am J Public Health 103: 1249-1254, 2013

2. Rejdak K, Petzold A, Lin L, Smith M, Kitchen N and Thompson E: Decreased CSF hypocretin-1 (orexin-A) after acute haemorrhagic brain injury. J Neurol Neurosurg Psychiatyr 76 597-598, 2005.

3. Gao XB and Wang AH: Experience-dependent plasticity in hypocretin/orexin neurons re-setting arousal threshold. Acta Physiol (Oxf) 198: 251-262, 2010.

4. de Lecea L: A decade of hypocretins: past, present and future of the neurobiology of arousal. Acta phyisiol (Oxf) 198: 203-208, 2010.

5. Peri CV, Shaffrey ME, Farace E, Cooper E, Alves WM, Cooper JB, Young JS, et al: Pilot study of electrical stimulation on median nerve in comatose severe brain injured patients: 3-month outcome. Brain Inj 15: 903-910, 2001.

6. Yokoyama T, Kamei T and Kanno T: Right median nerve stimulation for comatose patients. Soc Treat Coma 5: 117-125, 1996

7. Suzuki A, Nishimura H, Yoshioka K, et al: Electrical stimulation of median nerve in patients of prolonged coma. Soc Treat Coma 3: 75-85, 1994.

8. Moriya $\mathrm{T}$, et al: New therapeutic strategies for patients with unconsciousness and neurological deficits in acute stage with median nerve stimulation. Soc Treat Coma 7: 65-69, 1998.

9. Cooper JB, Jane JA, Alves WM and Cooper EB: Right median nerve electrical stimulation to hasten awakening from coma. Brain Inj 13: 261-267, 1999.

10. Feeney DM, Boyeson MG, Linn RT, Murray HM and Dail WG: Responses to cortical injury: I. Methodology and local effects of contusions in the rat. Brain Res 211: 67-77, 1981.

11. Stephens JR and Levy RH: Effects of valproate and citrulline on ammoniuminduced encephalopathy. Epilepsia 35: 164-171, 1994

12. Sakurai T, Amemiya A, Ishii M, Matsuzaki I, Chemelli RM, Tanaka H, Williams SC, et al: Orexins and orexin receptors: a family of hypothalamic neuropeptides and $G$ protein-coupled receptors that regulate feeding behavior. Cell 92: 573-585, 1998.

13. Randeva HS, Karteris E, Grammatopoulos D and Hillhouse EW: Expression of orexin-A and functional orexin type 2 receptors in the human adult adrenals: implications for adrenal function and energy homeostasis. J Clin Endocrinol Metab 86: 4808-4813, 2001 .

14. Williams G, Bing C, Cai XJ, Harrold JA, King PJ and Liu XH: The hypothalamus and the control of energy homeostasis: different circuits, different purposes. Physiol Behav 74: 683-701, 2001.
15. Smart D and Jerman J: The physiology and pharmacology of the orexins. Pharmacol Ther 94: 51-61, 2002.

16. Kukkonen JP, Holmqvist T, Ammoun S and Akerman KE: Functions of the orexigenic/hypocretinergic system. Am J Physiol Cell Physiol 283: C1567-C1591, 2002.

17. Taheri S, Ward H, Ghatei M and Bloom S: Role of orexins in sleep and arousal mechanisms. Lancet: 355: 847, 2000.

18. Samson WK, Gosnell B, Chang JK, Resch ZT and Murphy TC: Cardiovascular regulatory actions of the hypocretins in brain. Brain Res 831: 248-253, 1999.

19. Moreno-Balandrán E, Garzón M, Bódalo C, Reinoso-Suárez F and de Andrés I: Sleep-wakefulness effects after microinjections of hypocretin 1 (orexin A) in cholinoceptive areas of the cat oral pontine tegmentum. Eur J Neurosci 28: 331-341, 2008.

20. Peyron C, Tighe DK, van den Pol AN, de Lecea L, Heller HC, Sutcliffe JG and Kilduff TS: Neurons containing hypocretin (orexin) project to multiple neuronal systems. J Neurosci 18: 9996-10015, 1998

21. Vittoz NM, Schmeichel B and Berridge CW: Hypocretin/orexin preferentially activates caudomedial ventral tegmental area dopamine neurons. Eur J Neurosci 28: 1629-1640, 2008.

22. Sakurai T, Nagata R, Yamanaka A, Kawamura H, Tsujino N, Muraki Y, Kageyama $\mathrm{H}$, et al: Input of orexin/hypocretin neurons revealed by a genetically encoded tracer in mice. Neuron 46: 297-308, 2005.

23. Yoshida K, McCormack S, España RA, Crocker A and Scammell TE: Afferents to the orexin neurons of the rat brain. J Comp Neurol 494: 845-861, 2006.

24. Yamanaka A, Beuckmann CT, Willie JT, Hara J, Tsujino N, Mieda M, Tominaga M, et al: Hypothalamic orexin neurons regulate arousal according to energy balance in mice. Neuron 38: 701-713, 2003.

25. Cossu G: Therapeutic options to enhance coma arousal after traumatic brain injury: State of the art of current treatments to improve coma recovery. Br J Neurosurg 28: 187-198, 2014.

26. Cooper EB and Cooper JB: Electrical treatment of coma via the median nerve. Acta Neurochir Suppl 87: 7-10, 2003.

27. Liu JT, Wang CH, Chou IC, Sun SS, Koa CH and Cooper E: Regaining consciousness for prolonged comatose patients with right median nerve stimulation. Acta Neurochir Suppl 87: 11-14, 2003.

28. Cooper EB, Scherder EJ and Cooper JB: Electrical treatment of reduced consciousness: Experience with coma and Alzheimer's disease. Neuropsychol Rehabili 15: 389-405, 2005.

29. Liu JT, Lee JK, Tyan YS, Liu CY and Lin TB: Change in cerebral perfusion of patients with coma after treatment with right median nerve stimulation and hyperbaric oxygen. Neuromodulation 11: 296-301, 2008

30. Sauvage M and Steckler T: Detection of corticotrophin-releasing hormone receptor 1 immunoreactivity in cholinergic, dopaminergic and noradrenergic neurons of the murine basal forebrain and brainstem nuclei-potential implication for arousal and attention. Neuroscience 104: 643-652, 2001.

31. Kayama Y and Koyama Y: Brainstem neural mechanisms of sleep and wakefulness. Eur Urol 33: 12-15, 1998.

32. Butler MP, Rainbow MN, Rodriguez E, Lyon SM and Silver R: Twelve-hour days in the brain and behavior of split hamsters. Eur J Neurosci 36: 2556-2566, 2012.

33. Belle MD, Hughes AT, Bechtold DA, Cunningham P, Pierucci M, Burdakov D and Piggins HD: Acute suppressive and long-term phase modulation actions of orexin on the mammalian circadian clock. J Neurosci 34: 3607-3621, 2014.

34. Donjacour CE, Kalsbeek A, Overeem S, Lammers GJ, Pévet P, Bothorel B, Pijl H and Aziz NA: Altered circadian rhythm of melatonin concentrations in hypocretin-deficient men. Chronobio Int 29: 356-362, 2012.

35. Yan A, Zhang L, Tang Z, Zhang Y, Qin C, Li B, Li W and Lin H: Orange-spotted grouper (Epinephelus coioides) orexin: molecular cloning, tissue expression, ontogeny, daily rhythm and regulation of NPY gene expression. Peptides 32: 1363-1370, 2011. 